\title{
NORMA MORAL, NORMA SOCIAL SUBJETIVA Y ACTITUDES COMO PREDICTORES DE LA INTENCIÓN DE INICIAR RELACIONES SEXUALES EN LA ADOLESCENCIA
}

\author{
Pablo Alonso Sanabria Ferrad, Luis Artemo González Quevedo, Olga Paredes, Sandra Moreno \\ Facultad de Medicina, Universidad Militar Nueva Granada \\ Correspondencia: pablo.sanabria@unimilitar.edu.co \\ Recibido: Enero 14 de 2013 Aceptado: Abril 16 de 2013
}

\begin{abstract}
Resumen
La Teoría de la Acción Razonada (TAR) ha permitido identificar las variables que predicen la intención de los y las adolescentes para comenzar a tener relaciones sexuales, y se ha demostrado que la inclusión de la variable norma moral incrementa el poder explicativo de la TAR para las adolescentes mujeres, pero no se había establecido si ésto aplica a los hombres. Es por ello que este estudio pretendió, en primera instancia, corroborar la consistencia de la TAR y la inclusión de la variable norma moral, para predecir la intención de los y las adolescentes para iniciar sus relaciones sexuales, así como establecer si las variables que predicen la intención son las mismas para ambos sexos. Se tomó una muestra de 355 estudiantes voluntarios de tres colegios privados de Bogotá, Colombia, militar (33,5\%), laico $(32,7 \%)$ y religioso $(33,8 \%)$. Del total de la muestra el $52 \%$ fueron hombres y $48 \%$ mujeres. El $41 \%$ pertenecían al $9^{\circ}$ grado, $42 \%$ a $10^{\circ}$ grado y $17 \%$ al grado 11 . La edad promedio fue 15,33 años (DT $=1,03)$. Se les aplicó un cuestionario estructurado de autoinforme con 136 preguntas, previo consentimiento informado pasivo escrito de sus padres de familia. Para este artículo solamente se tuvieron en cuenta las variables: actitudes hacia las relaciones sexuales en la adolescencia, norma moral, norma social subjetiva (norma de padre y amigos) e intención de tener relaciones sexuales. Con el análisis de regresión múltiple se confirmó que la variable norma moral mejora el poder predictivo de la TAR para predecir la intención para iniciar a tener relaciones sexuales en la adolescencia, que la norma social subjetiva de los amigos es determinante en la intención de los hombres, mientras que para las mujeres es la norma social subjetiva de los padres. Los hallazgos son determinantes para el diseño de estrategias que permitan prevenir el embarazo adolescente.
\end{abstract}

Palabras clave: Relaciones sexuales, adolescencia, norma social, norma moral, actitudes, intención.

\section{MORAL STANDARD, SUBJECTIVE SOCIAL STANDARD AND ATTITUDES AS PREDICTIVE OF INTENT TO START SEX IN ADOLESCENCE}

\begin{abstract}
The Theory of Reasoned Action (TRA) has identified the variables that predict the intention of adolescents to start having sexual intercourse, and has been shown that the inclusion of moral norm variable increases the explanatory power of TRA for adolescent girls, but it had not been established if this applies to men. Therefore, this study aimed at first instance to check the consistency of the TRA and the inclusion of moral norm variable to predict the intention of adolescents to initiate sexual intercourse and to establish whether the variables that predict intention are the same for both sexes. A sample of 355 volunteer students from three private schools in Bogotá, Colombia was used: military $(33.5 \%)$ secular $(32.7 \%)$ and religious (33.8 \% of the total sample $52 \%$ were men and $48 \%$ female. $41 \%$ belonged to 9 th grade, $42 \%$ to $10^{\text {th }}$ grade and $17 \%$ to 11 th grade. The mean age was 15.33 years $(\mathrm{SD}=1.03)$. They answered a structured self-report questionnaire with 136 questions, previous
\end{abstract}


NORMA MORAL, NORMA SOCIAL SUBJETIVA Y ACTITUDES COMO PREDICTORES DE LA INTENCIÓN

DE INICIAR RELACIONES SEXUALES EN LA ADOLESCENCIA

passive informed consent of their parents. This article only took into account the variables: attitudes toward sexual intercourse in adolescence, moral norm, subjective social norm (norm of parents and friends) and intention to have intercourse. With multiple regression analysis, it was confirmed that the moral norm variable improves the predictive power of TRA to predict the intention to initiate sexual intercourse during adolescence, that subjective social norm of friends is determinant in the intention of men, while for women is the parental social subjective norm. The findings are critical for designing strategies to prevent teen pregnancy.

Key words: Sexual intercourse, adolescense, social norm, moral norm, attitudes, intention.

\title{
NORMA MORAL, NORMA SOCIAL SUBJETIVA E ATITUDES COMO PREDICTORES DA INTENÇÃO DE INICIAR RELAÇÕES SEXUAIS NA ADOLESCÊNCIA
}

\begin{abstract}
Resumo
A Teoria da Ação Razoada (TAR) tem permitido identificar as variáveis que predizem a intenção dos $e$ das adolescentes para começar a ter relações sexuais, e tem-se demonstrado que a inclusão da variável norma moral incrementa o poder explicativo da TAR para as adolescentes mulheres, mas não se tinha estabelecido se isto aplica aos homens. É por isso, que este estudo pretendeu, em primeira instância, corroborar a consistência da TAR e a inclusão da variável norma moral, para predizer a intenção dos e das adolescentes para iniciar as suas relações sexuais, assim como estabelecer se as variáveis que predizem a intenção são as mesmas para os dois sexos. Tomou-se uma amostra de 355 estudantes voluntários de três colégios privados de Bogotá, Colômbia, militar (33,5\%), laico (32,7\%) e religioso $(33,8 \%)$. Do total da amostra o $52 \%$ foram homens e $48 \%$ mulheres. O $41 \%$ pertenciam à $9^{\circ}$ série, $42 \%$ à $10^{\circ}$ série e $17 \%$ à série 11 . A idade média foi 15,33 anos (DT $\left.=1,03\right)$. Se lhes aplicou um questionário estruturado de autoinforme com 136 perguntas, prévio consentimento informado passivo escrito dos seus pais de família. Para este artigo somente se tiveram em conta as variáveis: atitudes para as relações sexuais na adolescência, norma moral, norma social subjetiva (norma de pai $e$ amigos) e intenção de ter relações sexuais. Com a análise de regressão múltipla se confirmou que a variável norma moral melhora o poder preditivo da TAR para predizer a intenção para começar a ter relações sexuais na adolescência, que a norma social subjetiva dos amigos é determinante na intenção dos homens, enquanto que para as mulheres é a norma social subjetiva dos pais. As descobertas são determinantes para o desenho de estratégias que permitam prevenir a gravidez adolescente.
\end{abstract}

Palavras chave: Relações sexuais, adolescência, norma social, norma moral, atitudes, intenção.

La Salud Sexual y Reproductiva (SSR) de los adolescentes se reconoce como un área prioritaria desde la Conferencia Internacional sobre Población y Desarrollo realizada en el Cairo en 1994 (1). A pesar de los esfuerzos realizados a nivel nacional e internacional, en los últimos veinte años la tasa de embarazo en adolescentes ha aumentado significativamente, especialmente entre las adolescentes latinoamericanas (2-4). De igual manera, ha disminuido la edad de inicio de las relaciones sexuales, todo ello pese a los programas de educación sexual que deberían llevar a los jóvenes a prevenir el embarazo y las conductas de riesgo. Aunque diversos factores se asocian con la iniciación temprana de la actividad sexual, actualmente no se tienen evidencias suficientes que confirmen que la norma moral mejora el poder predictivo de la TAR.

En Colombia, el análisis secundario de la información que arrojan las Encuestas de Demografía y Salud (4) revela que la proporción de adolescentes con hijos o embarazadas ha venido aumentando en la última década: en 1990, el 12.8\% de las adolescentes eran ya madres o estaban embarazadas, mientras que en el 2005 tal proporción alcanza el 20.5\%. Aunque ser madre a los 15 años es raro, tal condición se duplica entre las adolescentes de 15 años (3\% en 1990 y 6.5\% 
en 2005). Tan importante como la proporción de madres adolescentes, es la edad a la cual inician esa maternidad. Las adolescentes inician su maternidad cada vez más temprano. La proporción de madres adolescentes que tuvo su hijo antes de los 18 años pasa de $7 \%$ en 1990 a $11 \%$ en el 2000 . Así, no solo la proporción de adolescentes con hijos se ha incrementado, sino que la edad a la cual tienen ese primer hijo ha venido disminuyendo. El aumento en la tasa de las jóvenes que inician su actividad sexual a más temprana edad, está relacionado con una mayor exposición al riesgo de embarazo.

Con respecto a la exposición al riesgo de embarazo, el análisis de Flórez, señala que la proporción de adolescentes con actividad sexual se ha duplicado en la última década, al pasar de $21 \%$ en 1990 a $44 \%$ en el 2005 (4). La edad a la cual las jóvenes inician sus relaciones sexuales ha disminuido notablemente y la proporción que ha tenido relaciones sexuales a cada edad ha aumentado. Por ello, no sólo la incidencia de las relaciones sexuales es mayor, sino que la edad de inicio se ha acelerado: en 1990, el 5\% de las adolescentes había iniciado relaciones sexuales antes de los 15 años; en el 2005, tal porcentaje casi se triplica, llegando al $13.7 \%$.

Estos datos justifican que en Colombia la SSR de los y las adolescentes se haya definido como una de las diez prioridades en Salud Pública en el Plan Nacional de Salud 2007 - 2010 (5). Igualmente, explican que en los últimos años se haya generado un creciente interés por el estudio de la edad de inicio de la actividad sexual en la adolescencia. De acuerdo con diversos autores la edad a la que ocurre las relaciones sexuales penetrativas es el determinante más próximo de varios problemas de SSR, entre otros (6-17).

La importancia de la edad de inicio de relaciones sexuales se constata al revisar los hallazgos de los estudios que indican que entre más temprano comiencen los adolescentes su actividad sexual, es menos probable que hayan desarrollado las competencias requeridas para manejar las exigencias de las relaciones sexuales, así como sus posibles implicaciones. Efectivamente, en los informes de investigación más recientes se observa la tendencia de los investigadores a reconocer que aunque la exploración sexual es un evento que ocurre habitualmente en la adolescencia, el inicio de relaciones sexuales antes de los 16 años puede ser problemático $(12,13,18,19)$. Según Smith los cambios físicos, emocionales, cognitivos y sociales que ocurren entre los 11 y los 15 años de edad pueden interferir con la habilidad de los adolescentes para tomar decisiones sexuales autónomas (15).

Entre los modelos más populares que intentan explicar por qué las personas eligen involucrarse en comportamientos que afectan su salud son los denominados Modelos Cognitivos Sociales (MCSs). Los MCSs plantean que la intención de realizar un comportamiento es el mejor predictor de que éste se ejecute. Estos modelos explican la intención a partir de variables cognitivas, es decir, procesos mentales que intervienen entre los estímulos observables y las respuestas de las personas en las situaciones de la vida diaria (20).

Entre las cogniciones que se han considerado para predecir la intención conductual, encontramos la actitud personal y la norma subjetiva (21), las expectativas de la situación, las expectativas de resultados y las expectativas de autoeficacia (22). De acuerdo con estos MCSs el comportamiento es el resultado de un proceso de toma de decisiones basado en un análisis subjetivo, deliberado y sistemático de la información que la persona tiene disponible. No obstante, este proceso de toma de decisiones no es algo que ocurre cada vez que la persona enfrenta una situación ante la cual debe optar por una u otra alternativa de acción; estos modelos asumen que el resultado del análisis se mantiene en la memoria y es recuperado automáticamente cuando es necesario resolver un conflicto o tomar una decisión (23).

De todos los MCSs disponibles, uno de los más citados en la literatura es el de la TAR $(21,24,25)$. La TAR, al igual que los otros MCSs, asume que las personas son razonables y que hacen uso sistemático de la información para decidir de qué manera debe comportarse o dejar de actuar. En esta teoría se asume que el comportamiento está orientado a un fin y que las personas eligen aquellas acciones que les permiten lograr lo que se proponen. Para la TAR la intención es el determinante inmediato del comportamiento. A su vez la intención está determinada por dos factores: el primero es la actitud personal hacia el comportamiento y el segundo es la percepción de la presión social o norma social subjetiva, para llevar a cabo o no la acción. Así, la actitud hacia el comportamiento está determinada por la creencia de que dicho comportamiento producirá resultados esperados valorados de forma positiva o negativa. A su vez, la norma social 
subjetiva está determinada por la valoración que hace el individuo del comportamiento y por la motivación para ajustarse a las normas percibidas. Por lo tanto, de acuerdo con la TAR la predicción del estatus sexual de los y las adolescentes estaría determinada por la intención de tener relaciones sexuales, que a su vez estaría en función del peso relativo que el o la joven le dé a su actitudes hacia el inicio y la importancia de la norma social subjetiva. Además de los dos componentes considerados (26) señalan que la inclusión de la norma moral en la TAR, podría contribuir de forma importante en el incremento del potencial explicativo del modelo. Los resultados del estudio de (27) son consistentes con este planteamiento. Ahora bien, estudios como el de (28) han encontrado que la norma social subjetiva tiene un efecto más fuerte en la intención de tener relaciones sexuales que las actitudes personales. El indicador de la norma social subjetiva más citado en la literatura es la prevalencia percibida de actividad sexual en el grupo de iguales. Numerosos estudios que han incluido este indicador, han mostrado su asociación significativa con la edad de inicio de relaciones sexuales $(29,29-34)$, la disposición para comenzar actividad sexual penetrativa en la adolescencia $(32,34)$ y el estatus sexual $(32,34)$.

La tendencia a sobrestimar el comportamiento del grupo de pares ha sido objeto de diversos estudios. Por ejemplo, en un estudio se encontró que los estudiantes sobrestiman los niveles de actividad sexual de sus pares, el número de parejas, la incidencia de las infecciones de transmisión sexual y los índices de embarazos no deseados (35). También se observó que, por el contrario, los estudiantes subestiman la frecuencia de uso del condón por parte de sus pares. Los únicos índices estimados con exactitud corresponden a la toma de la prueba de VIH. En otro estudio, que tenía como objetivo comparar la percepción de la norma de pares en el consumo de alcohol, drogas y comportamiento sexual con el comportamiento actual y determinar si existe relación entre la percepción de comportamiento normativo y el comportamiento actual (real) del estudiante (36), se encontró que se tendía a malinterpretar la norma social subjetiva de todos los comportamientos mencionados, dado que la mayoría de los estudiantes sobreestimaron el comportamiento normativo (especialmente para el número de parejas y las relaciones vaginales). Los datos indicaron una relación significativa entre el comportamiento del estudiante y la percepción de la norma de comportamiento, es decir, aquellos que se comprometían en alguna conducta fueron aquellos que más probablemente la veían como normativa.

Resultados como los que se acaban de exponer justifican el que las intervenciones basadas en la teoría de la norma social subjetiva, que apuntan a prevenir los comportamientos de riesgo en estudiantes, sean cada vez más populares (37). Se asume que esas intervenciones modifican las falsas valoraciones del estudiante en cuanto a los comportamientos de consumo de sus pares y que de esta manera se generan cambios en el comportamiento de consumo individual. Ahora bien, aunque evidentemente el cambio en la norma social subjetiva puede tener un efecto en los comportamientos que determinan la salud sexual de las personas, los datos disponibles sugieren que es necesario conocer $e$ intervenir otros determinantes del comportamiento sexual, entre ellos las emociones y el desarrollo del razonamiento moral, especialmente los que se relacionan con el contexto social. Es de gran importancia modificar normas sociales para apoyar el cambio y el mantenimiento del comportamiento, y para abordar los factores estructurales que contribuyen al comportamiento sexual de riesgo particularmente (38).

Ahora bien, aunque la TAR ha contribuido de forma consistente con la identificación de las variables que permiten predecir la intención de los y las adolescentes para comenzar a tener relaciones sexuales, y se ha demostrado que la inclusión de la norma moral incrementa el poder explicativo del modelo en el caso de adolescentes mujeres, aún no se ha establecido si la fortaleza de este último modelo también se mantiene en los adolescentes hombres. Es por ello que el presente estudio pretende en primera instancia corroborar la consistencia de la TAR y la inclusión de la variable norma moral, para predecir la intención de los y las adolescentes para iniciar a tener relaciones sexuales, así como establecer si las variables que predicen la intención son las mismas tanto para hombres como para mujeres.

\section{Metodología}

\section{Tipo de estudio}

Se realizó un estudio de corte transversal, correlacional multivariado. 


\section{Participantes}

Para el propósito de este estudio se contó con la participación voluntaria de 355 estudiantes de tres colegios privados de la ciudad de Bogotá, Colombia: militar $(33,5 \%)$, laico $(32,7 \%)$ y religioso $(33,8 \%)$. Del total de la muestra el $52 \%$ fueron hombres y $48 \%$ mujeres. El $41 \%$ pertenecían al $9^{\circ}$ grado, $42 \%$ a $10^{\circ}$ grado y $17 \%$ a grado 11 . La edad promedio fue 15,33 años $(\mathrm{DT}=1,03)$.

\section{Instrumentos y medidas}

Para la obtención de información se utilizó un cuestionario estructurado de autoinforme que incluía en total 136 preguntas. Sin embargo, para este artículo solamente se tuvo en cuenta la información sobre las siguientes variables:

Intención de tener relaciones sexuales en la adolescencia: Se evaluó a partir de una escala constituida por dos afirmaciones ("A mí me gustaría comenzar a tener relaciones sexuales en este momento de mi vida"; "A mí me gustaría tener relaciones sexuales después de casarme") frente a las que el o la joven indicaba su grado de acuerdo, utilizando una escala Likert de 5 puntos, en donde 1 era Totalmente en desacuerdo y 5 era Totalmente de acuerdo. La confiabilidad de esta escala es de $\alpha=0,68$. Los puntajes altos indican mayor intención de tener relaciones sexuales en la adolescencia.

Norma social subjetiva: Se utilizaron los ítems desarrollados por García-Díaz (27) para obtener información sobre los siguientes indicadores: a) La percepción que tienen las y los adolescentes de la actitud de las personas significativas (padres y amigos) hacia tener relaciones sexuales en la adolescencia. Para evaluar dicha actitud percibida de las y los adolescentes, se construyeron 2 sub-escalas tipo Likert de cinco puntos, compuesta por tres ítems la de los padres $(\alpha=0,68)$ y 5 la de los amigos $(\alpha=0,74)$. En cada una de ellas se le preguntó a las y los adolescentes acerca de lo que cree respecto a la aprobación o no, por parte de las personas significativas, de que ella o él tuvieran relaciones sexuales a su edad, de que tuviera relaciones sexuales pre-matrimoniales y de que tuviera relaciones sexuales durante la adolescencia. En cada una de estas sub-escalas los puntajes altos indican una percepción de una actitud favorable hacia las relaciones sexuales en la adolescencia por parte de padres y amigos. b) La norma de pares percibida (prevalencia percibida de actividad sexual en el grupo de adolescentes), que se evaluó por medio de dos ítems con respuestas escala tipo Likert de cinco puntos, los cuales preguntaban acerca de la creencia de las y los adolescentes sobre el número de amigas y amigos que tenían relaciones sexuales. Los puntajes altos en esta escala indican mayor prevalencia percibida de actividad sexual, tanto en el grupo de compañeros y compañeras, como en el grupo de amigos y amigas.

Actitudes hacia las relaciones sexuales: Se evaluó a partir de dos ítems utilizados en estudios previos de $(39,40)$ para indagar el nivel de acuerdo de los y las jóvenes con las relaciones sexuales en la adolescencia. Para responder se les proponía una escala tipo Likert en la que 1 era totalmente en desacuerdo y 5 totalmente de acuerdo. Su nivel de confiabilidad es de $\alpha=0,94$.

Norma de pares: Se utilizaron dos ítems, en donde las y los jóvenes debían indicar el porcentaje de amigos y amigas que ellos creían habían comenzado a tener relaciones sexuales, en una escala de $0 \%$ a $100 \%$.

Norma moral: Se usó la escala diseñada por GarcíaDíaz (27) compuesta por seis ítems con una escala de respuesta tipo Likert de cinco puntos para establecer cómo creen que se siente una persona cuando tiene relaciones sexuales en la adolescencia (por ejemplo, "se siente avergonzada", "siente que está haciendo algo malo", "se siente culpable"). Los puntajes altos en esta escala indican que el hecho de tener relaciones sexuales en la adolescencia es valorado como un comportamiento moralmente aceptable. Su nivel de confiabilidad es de $\alpha=0.82$.

\section{Procedimiento}

Se seleccionaron tres colegios cuyo proyecto educativo institucional, fuera parte de una concepción religiosa, militar y laica. Se seleccionaron los estudiantes con las características mencionadas en la sección de participantes y se solicitó consentimiento informado pasivo por escrito, tanto de padres de familia como de los y las adolescentes. La aplicación de los cuestionarios se hizo grupalmente con la orientación de los investigadores aplicando la totalidad del instrumento en una sesión de una hora. 


\section{Resultados}

\section{Análisis descriptivo}

En la tabla 1 se presentan los estadísticos descriptivos para las variables del estudio. Los análisis de comparaciones de medias mostraron diferencias significativas entre hombres y mujeres para las variables evaluadas excepto en la variable norma de pares (percepción que tienen las y los jóvenes del porcentaje de adolescentes que han tenido relaciones sexuales). La tendencia general es que los hombres presentan puntuaciones más altas que las mujeres.

\section{Análisis de correlación}

Con el fin de establecer la relación entre la intención de los y las adolescentes para iniciar las relaciones sexuales en la actualidad y las variables predictoras, se realizó un análisis de correlación de Pearson, encontrándose una correlación significativa y positiva para todas las variables predictoras. Sólo la variable norma de pares (percepción del porcentaje de amigos y amigas que ya han tenido relaciones sexuales) presenta una correlación relativamente más baja en comparación con las demás variables (ver tabla 2 ).

Tabla 1. Estadísticos descriptivos y diferencia de medias para las variables del estudio

\begin{tabular}{|c|c|c|c|c|c|}
\hline Variables & Sexo & $\mathbf{N}$ & Media & Desviación típ. & Valores $\mathbf{t}$ \\
\hline \multirow{2}{*}{ Intención relaciones sexuales } & Hombre & 176 & 3.50 & 1.14 & \multirow{2}{*}{$7.81 * *$} \\
\hline & Mujer & 157 & 2.54 & 1.11 & \\
\hline \multirow{2}{*}{ Norma social subjetiva } & Hombre & 183 & 3.32 & 0.74 & \multirow{2}{*}{$7.84 * *$} \\
\hline & Mujer & 166 & 2.71 & 0.69 & \\
\hline \multirow{2}{*}{ Norma - Padres } & Hombre & 182 & 3.00 & 0.98 & \multirow{2}{*}{$6.66 * *$} \\
\hline & Mujer & 166 & 2.32 & 0.93 & \\
\hline \multirow{2}{*}{ Norma - Amigos } & Hombre & 182 & 3.62 & 0.79 & \multirow{2}{*}{$6.06 * *$} \\
\hline & Mujer & 166 & 3.11 & 0.79 & \\
\hline \multirow{2}{*}{$\begin{array}{l}\text { Actitudes hacia las relaciones } \\
\text { sexuales }\end{array}$} & Hombre & 185 & 3.21 & 1.03 & \multirow{2}{*}{$5.54 * *$} \\
\hline & Mujer & 166 & 2.59 & 1.04 & \\
\hline \multirow{2}{*}{ Norma de pares } & Hombre & 182 & 37.12 & 23.84 & \multirow{2}{*}{1.51} \\
\hline & Mujer & 165 & 33.09 & 25.80 & \\
\hline \multirow{2}{*}{ Norma moral } & Hombre & 184 & 3.54 & 0.74 & \multirow{2}{*}{$6.47 * *$} \\
\hline & Mujer & 166 & 3.01 & 0.79 & \\
\hline
\end{tabular}

Nota. $* * p<0.01$

Tabla 2. Matriz de correlación de Pearson entre la intención de iniciar relaciones sexuales y sus variables predictoras

\begin{tabular}{|c|c|c|c|c|c|c|c|}
\hline Variables & 1 & 2 & 3 & 4 & 5 & 6 & 7 \\
\hline 1. Intención relaciones sexuales & 1.000 & $.623 *$ & $.539 *$ & $.499 *$ & $.655^{*}$ & $.362 *$ & $.598^{*}$ \\
\hline 2. Norma social subjetiva & & 1.000 & $.872 *$ & $.802 *$ & $.621 *$ & $.441^{*}$ & $.612^{*}$ \\
\hline 3. Norma de Padres & & & 1.000 & $.403 *$ & $.543^{*}$ & $.319 *$ & $.569 *$ \\
\hline 4. Norma de Amigos & & & & 1.000 & $.500 *$ & $.427 *$ & $.451^{*}$ \\
\hline $\begin{array}{l}\text { 5. Actitud hacia las relaciones } \\
\text { sexuales }\end{array}$ & & & & & 1.000 & $.447^{*}$ & $.604 *$ \\
\hline 6. Norma de pares & & & & & & 1.000 & $.300 *$ \\
\hline 7. Norma moral & & & & & & & 1.000 \\
\hline
\end{tabular}

$* p<0.05$ 


\section{Análisis de regresión lineal múltiple}

Para responder a la pregunta de investigación de si la TAR predice la intención de los y las adolescentes a iniciar sus relaciones sexuales, se realizó un análisis de regresión múltiple jerárquica hacia atrás, en donde en un primer paso se incluyen todas las variables que conforman el modelo y se van descartando aquellas variables que no aportan en la predicción de la intención.

Para la primera parte del análisis se incluyeron tanto los hombres como las mujeres y se probó la TAR, sin la inclusión de la variable norma moral. En la segunda parte del análisis, se incluyó la variable norma moral, con el fin de confirmar si dicha variable mejora la predicción de la TAR. La tercera parte del análisis, se prueba la TAR sin y con la variable norma moral de forma separada para los y las jóvenes, con el fin de establecer, en primer lugar, si la TAR predice de igual forma la intención de hombres y mujeres, y si la variable norma moral mejora la predicción en ambos sexos. Los resultados se describen a continuación.
En la tabla 3a se observa que para el caso de la muestra general de los y las jóvenes sin la variable norma moral, se encuentra que el modelo obtenido llega a explicar un $50,3 \%$ de la varianza total. Las variables incluidas en el modelo son: actitudes positivas hacia las relaciones sexuales, norma de amigos y norma de padres de que se deben tener relaciones sexuales (ver tabla 3b).

En la tabla $3 b$ indica que al incluir la variable norma moral dentro del análisis, el modelo permite explicar el $52,8 \%$ de la varianza, mejorando en un $2.5 \%$ el poder predictivo del TAR. El modelo final incluye las variables: actitudes positivas hacia las relaciones sexuales, norma moral a favor de las relaciones sexuales, así como norma de amigos y padres a favor de las mismas (ver tabla 4b).

Es importante anotar que de acuerdo con el estadístico FIV, no existe colinealidad para ninguna de las variables evaluadas, ya que su valor es menor a 5 (ver tablas 4a y 4b).

Tabla 3. Resumen del modelo de regresión para la muestra de los y las jóvenes.

a. Sin la variable norma moral

\begin{tabular}{ccccc}
\hline Modelo & $\mathrm{R}$ & $\mathrm{R}$ cuadrado & R cuadrado corregida & Error típ. de la estimación \\
\hline 2 & .713 & .508 & .503 & .8640 \\
\hline \multicolumn{7}{l}{ b. Con la variable norma moral } \\
\hline Modelo & $\mathrm{R}$ & $\mathrm{R}$ cuadrado & $\mathrm{R}$ cuadrado corregida & Error típ. de la estimación \\
\hline 2 & .731 & .534 & .528 & .8424 \\
\hline
\end{tabular}

Tabla 4. Coeficientes de regresión para la muestra de los y las jóvenes.

a. Sin la variable norma moral

\begin{tabular}{|c|c|c|c|c|c|c|c|c|c|c|c|}
\hline & \multirow{2}{*}{ Modelo } & \multicolumn{2}{|c|}{$\begin{array}{l}\text { Coeficientes no } \\
\text { estandarizados }\end{array}$} & \multirow{2}{*}{$\begin{array}{c}\begin{array}{c}\text { Coeficientes } \\
\text { estandarizados }\end{array} \\
\text { Beta }\end{array}$} & \multirow{2}{*}{$\mathrm{t}$} & \multirow{2}{*}{ Sig. } & \multicolumn{3}{|c|}{ Correlaciones } & \multicolumn{2}{|c|}{$\begin{array}{l}\text { Estadísticos de } \\
\text { colinealidad }\end{array}$} \\
\hline & & B & $\begin{array}{l}\text { Error } \\
\text { típ. }\end{array}$ & & & & $\begin{array}{l}\text { Orden } \\
\text { cero }\end{array}$ & Parcial & Semiparcial & Tolerancia & FIV \\
\hline \multirow{4}{*}{2} & (Constante) & -.048 & .205 & & -.234 & .815 & & & & & \\
\hline & Norma - Padres & .258 & .057 & .213 & 4.506 & .000 & .535 & .243 & .176 & .682 & 1.467 \\
\hline & Norma - Amigos & .278 & .066 & .191 & 4.186 & .000 & .500 & .227 & .163 & .729 & 1.371 \\
\hline & $\begin{array}{l}\text { Actitud relacio- } \\
\text { nes sexuales }\end{array}$ & .504 & .056 & .451 & 9.023 & .000 & .662 & .449 & .352 & .611 & 1.638 \\
\hline
\end{tabular}


b. Con la variable norma moral

\begin{tabular}{|c|c|c|c|c|c|c|c|c|c|c|c|}
\hline \multirow{2}{*}{\multicolumn{2}{|c|}{ Modelo }} & \multicolumn{2}{|c|}{$\begin{array}{l}\text { Coeficientes no } \\
\text { estandarizados }\end{array}$} & \multirow{2}{*}{$\begin{array}{l}\begin{array}{l}\text { Coeficientes } \\
\text { estandarizados }\end{array} \\
\text { Beta }\end{array}$} & \multirow{2}{*}{$t$} & \multirow{2}{*}{ Sig. } & \multicolumn{3}{|c|}{ Correlaciones } & \multicolumn{2}{|c|}{$\begin{array}{l}\text { Estadísticos de } \\
\text { colinealidad }\end{array}$} \\
\hline & & B & $\begin{array}{l}\text { Error } \\
\text { típ. }\end{array}$ & & & & $\begin{array}{l}\text { Orden } \\
\text { cero }\end{array}$ & Parcial & Semiparcial & Tolerancia & FIV \\
\hline \multirow{5}{*}{2} & (Constante) & -.500 & .227 & & -2.21 & .028 & & & & & \\
\hline & Norma - Padres & .174 & .059 & .144 & 2.940 & .004 & .535 & .162 & .112 & .605 & 1.653 \\
\hline & Norma - Amigos & .236 & .066 & .162 & 3.600 & .000 & .500 & .197 & .137 & .712 & 1.404 \\
\hline & $\begin{array}{l}\text { Actitud relacio- } \\
\text { nes sexuales }\end{array}$ & .412 & .059 & .368 & 7.023 & .000 & .662 & .364 & .267 & .526 & 1.901 \\
\hline & Norma moral & .331 & .078 & .220 & 4.217 & .000 & .599 & .229 & .160 & .533 & 1.876 \\
\hline
\end{tabular}

El siguiente paso fue establecer si el modelo cambia según se trate de hombres o mujeres, y si la variable norma moral aporta al modelo predictivo en ambos sexos. Para ello se realizó el análisis de regresión múltiple jerárquica hacia atrás segregado según el sexo de los y las participantes (ver tablas 5 a 8).

En la tabla 6a, se observa que al realizar el análisis de regresión para la muestra de hombres, sin la variable norma moral, el modelo explica el $51,6 \%$ de la varianza observada (ver tabla 5a), y que las variables incluidas dentro del modelo son: la actitud favorable hacia relaciones sexuales y la norma de padres y amigos a favor de la relaciones sexuales en la adolescencia (ver tabla 6a).

Al incluir la variable norma moral, se observa que el modelo permite explicar el $53.8 \%$ de la varianza, aumentando en un $2,2 \%$ su poder predictivo (ver tabla $5 b$ ). El modelo final incluye las variables: actitud favorable hacia las relaciones sexuales en la adolescencia, norma moral y norma de amigos a favor de las relaciones sexuales. En este caso, la variable norma de padres no aporta al modelo (ver tabla 6b).

Es importante también anotar que de acuerdo con el estadístico FIV, se puede afirmar que no existe colinealidad para ninguna de las variables evaluadas, ya que su valor es menor a 5 (ver tablas $6 a$ y $6 b$ ).

En la tabla 7a, se observa que para las mujeres el modelo sin la variable norma moral llega a explicar $38,6 \%$ de la varianza observada. En el modelo final se concluyen las variables: actitud a favor hacia las relaciones y una norma de padres también a favor (ver tabla 8a).

Al incluir la variable norma moral, su predicción se incrementa al 40,4\%, mejorando en un $1,8 \%$ (ver tabla 7b). El modelo final incluye las variables: actitud a favor de las relaciones sexuales, norma de padres, y norma moral también a favor (ver tabla 8b).

Tabla 5. Resumen del modelo de regresión para los hombres.

a. Sin la variable norma moral

\begin{tabular}{ccccc}
\hline \multirow{2}{*}{ Modelo } & \multicolumn{5}{c}{$\mathrm{R}$} \\
\cline { 2 - 5 } & Sexo $=$ Hombre (Seleccionado) & R cuadrado & R cuadrado corregida & Error típ. de la estimación \\
\hline 2 & .718 & .516 & .507 & .8063 \\
\hline \multirow{2}{*}{ b. Con la variable norma moral } & & & \\
\hline \multirow{2}{*}{ Modelo } & \multicolumn{5}{c}{$\mathrm{R}$} & \\
\cline { 2 - 5 } & Sexo $=$ Hombre (Seleccionado) & R cuadrado & R cuadrado corregida & Error típ. de la estimación \\
\hline 3 & .734 & .538 & .530 & .7870 \\
\hline
\end{tabular}


Tabla 6. Coeficientes de regresión para la muestra de hombres.

a. Sin la variable norma moral

\begin{tabular}{|c|c|c|c|c|c|c|c|c|c|c|c|}
\hline & \multirow{2}{*}{ Modelo } & \multicolumn{2}{|c|}{$\begin{array}{l}\text { Coeficientes no } \\
\text { estandarizados }\end{array}$} & \multirow{2}{*}{$\begin{array}{c}\begin{array}{c}\text { Coeficientes } \\
\text { estandarizados }\end{array} \\
\text { Beta }\end{array}$} & \multirow{2}{*}{$\mathrm{t}$} & \multirow{2}{*}{ Sig. } & \multicolumn{3}{|c|}{ Correlaciones } & \multicolumn{2}{|c|}{$\begin{array}{c}\text { Estadísticos de coli- } \\
\text { nealidad }\end{array}$} \\
\hline & & B & Error típ. & & & & $\begin{array}{l}\text { Orden } \\
\text { cero }\end{array}$ & Parcial & Semiparcial & Tolerancia & FIV \\
\hline \multirow{4}{*}{2} & (Constante) & .001 & .300 & & .002 & .998 & & & & & \\
\hline & Norma - Padres & .175 & .076 & .149 & 2.300 & .023 & .489 & .176 & .124 & .699 & 1.432 \\
\hline & Norma - Amigos & .397 & .088 & .279 & 4.491 & .000 & .546 & .329 & .243 & .754 & 1.327 \\
\hline & $\begin{array}{l}\text { Actitud relacio- } \\
\text { nes sexuales }\end{array}$ & .482 & .074 & .443 & 6.499 & .000 & .655 & .450 & .351 & .628 & 1.593 \\
\hline
\end{tabular}

b. Con la variable norma moral

\begin{tabular}{|c|c|c|c|c|c|c|c|c|c|c|c|}
\hline & \multirow{2}{*}{ Modelo } & \multicolumn{2}{|c|}{$\begin{array}{l}\text { Coeficientes no } \\
\text { estandarizados }\end{array}$} & \multirow{2}{*}{$\begin{array}{c}\begin{array}{c}\text { Coeficientes } \\
\text { estandarizados }\end{array} \\
\text { Beta }\end{array}$} & \multirow{2}{*}{$\mathrm{t}$} & \multirow{2}{*}{ Sig. } & \multicolumn{3}{|c|}{ Correlaciones } & \multicolumn{2}{|c|}{$\begin{array}{c}\text { Estadísticos de coli- } \\
\text { nealidad }\end{array}$} \\
\hline & & B & Error típ. & & & & $\begin{array}{c}\text { Orden } \\
\text { cero }\end{array}$ & Parcial & Semiparcial & Tolerancia & FIV \\
\hline \multirow{4}{*}{3} & (Constante) & -.437 & .328 & & -1.33 & .184 & & & & & \\
\hline & Norma - Amigos & .320 & .090 & .225 & 3.540 & .001 & .546 & .265 & .187 & .690 & 1.450 \\
\hline & $\begin{array}{l}\text { Actitud relacio- } \\
\text { nes sexuales }\end{array}$ & .451 & .071 & .414 & 6.326 & .000 & .655 & .441 & .334 & .649 & 1.540 \\
\hline & Norma moral & .380 & .102 & .247 & 3.714 & .000 & .585 & .277 & .196 & .628 & 1.592 \\
\hline
\end{tabular}

Tabla 7. Resumen del modelo de regresión para las mujeres.

a. Sin la variable norma moral

\begin{tabular}{ccccc}
\hline \multirow{2}{*}{ Modelo } & \multicolumn{3}{c}{$\mathrm{R}$} \\
\cline { 2 - 5 } & Sexo $=$ Mujer (Seleccionado) & R cuadrado & R cuadrado corregida & Error típ. de la estimación \\
\hline 3 & .621 & .386 & .378 & .8755 \\
\hline
\end{tabular}

b. Con la variable norma moral

\begin{tabular}{ccccc}
\hline \multirow{2}{*}{ Modelo } & \multicolumn{4}{c}{$\mathrm{R}$} \\
\cline { 2 - 5 } & Sexo $=$ Mujer (Seleccionado) & R cuadrado & R cuadrado corregida & Error típ. de la estimación \\
\hline 3 & .636 & .404 & .393 & .8653 \\
\hline
\end{tabular}

Tabla 8. Coeficientes de regresión para la muestra de mujeres.

a. Sin la variable norma moral

\begin{tabular}{|c|c|c|c|c|c|c|c|c|c|c|c|}
\hline & \multirow{2}{*}{ Modelo } & \multicolumn{2}{|c|}{$\begin{array}{l}\text { Coeficientes no } \\
\text { estandarizados }\end{array}$} & \multirow{2}{*}{$\begin{array}{c}\begin{array}{c}\text { Coeficientes } \\
\text { estandarizados }\end{array} \\
\text { Beta }\end{array}$} & \multirow{2}{*}{$\mathrm{t}$} & \multirow{2}{*}{ Sig. } & \multicolumn{3}{|c|}{ Correlaciones } & \multicolumn{2}{|c|}{$\begin{array}{c}\text { Estadísticos de } \\
\text { colinealidad }\end{array}$} \\
\hline & & B & Error típ. & & & & $\begin{array}{l}\text { Orden } \\
\text { cero }\end{array}$ & Parcial & Semiparcial & Tolerancia & FIV \\
\hline \multirow{3}{*}{3} & (Constante) & .602 & .214 & & 2.814 & .006 & & & & & \\
\hline & Norma - Padres & .263 & .084 & .222 & 3.118 & .002 & .447 & .244 & .197 & .787 & 1.271 \\
\hline & $\begin{array}{l}\text { Actitud relacio- } \\
\text { nes sexuales }\end{array}$ & .515 & .075 & .487 & 6.836 & .000 & .589 & .483 & .432 & .787 & 1.271 \\
\hline
\end{tabular}


NORMA MORAL, NORMA SOCIAL SUBJETIVA Y ACTITUDES COMO PREDICTORES DE LA INTENCIÓN

DE INICIAR RELACIONES SEXUALES EN LA ADOLESCENCIA

b. Con la variable norma moral

\begin{tabular}{|c|c|c|c|c|c|c|c|c|c|c|c|}
\hline & \multirow{2}{*}{ Modelo } & \multicolumn{2}{|c|}{$\begin{array}{l}\text { Coeficientes no } \\
\text { estandarizados }\end{array}$} & \multirow{2}{*}{$\begin{array}{c}\begin{array}{c}\text { Coeficientes } \\
\text { estandarizados }\end{array} \\
\text { Beta }\end{array}$} & \multirow{2}{*}{$\mathrm{t}$} & \multirow{2}{*}{ Sig. } & \multicolumn{3}{|c|}{ Correlaciones } & \multicolumn{2}{|c|}{$\begin{array}{c}\text { Estadísticos de } \\
\text { colinealidad }\end{array}$} \\
\hline & & B & Error típ. & & & & $\begin{array}{l}\text { Orden } \\
\text { cero }\end{array}$ & Parcial & Semiparcial & Tolerancia & FIV \\
\hline \multirow{4}{*}{3} & (Constante) & .220 & .276 & & .798 & .426 & & & & & \\
\hline & Norma - Padres & .213 & .086 & .180 & 2.470 & .015 & .447 & .196 & .154 & .732 & 1.367 \\
\hline & $\begin{array}{l}\text { Actitud relacio- } \\
\text { nes sexuales }\end{array}$ & .424 & .086 & .400 & 4.947 & .000 & .589 & .371 & .309 & .595 & 1.681 \\
\hline & Norma moral & .244 & .113 & .175 & 2.163 & .032 & .501 & .172 & .135 & .592 & 1.689 \\
\hline
\end{tabular}

\section{Discusión}

El propósito de este estudio fue identificar si la TAR predice la intención de los y las adolescentes de tener relaciones sexuales, corroborar si la inclusión de la variable norma moral mejora el poder predictivo del modelo y si las estas variables predicen la intención tanto de hombres como de mujeres.

Coherentemente con estudios previos (26-33) los resultados revelan que la TAR predice un poco más de la mitad de la variabilidad de la intención de los y las adolescentes para empezar a tener relaciones sexuales.

Adicionalmente, los resultados apoyan las conclusiones del estudio de García-Díaz (27), en el sentido que la inclusión de la variable norma moral mejora el poder predictivo de la TAR, para pronosticar la intención de hombres y mujeres de tener relaciones sexuales en la adolescencia. Ésto implica que la percepción que tengan los y las adolescentes sobre cómo se siente (avergonzado, culpable, que está haciendo algo malo, etc.) cuando tiene relaciones coitales, aumenta el poder predictivo que la norma social subjetiva tiene para predecir el estatus sexual en la adolescencia.

Los hallazgos también permiten afirmar, que existen similitudes y diferencias importantes en las variables que predicen la intención de inicio de las relaciones sexuales entre hombres y mujeres. Las similitudes radican en que las variables que permiten predecir la intención de las relaciones sexuales tanto en hombres como en mujeres, son en su orden: las actitudes hacia las relaciones sexuales, la norma moral y la norma social subjetiva. Pero la diferencia está en que la actitud hacia las relaciones sexuales para hombres y mujeres, tiene que ver con la percepción de los o las jóvenes en relación a qué tan importante es que los adolescentes de su mismo sexo tengan relaciones sexuales, no así lo que ellas o ellos creen que están pensando los pares del sexo opuesto. Por otra parte, la norma social subjetiva, para el caso de las mujeres, está referida a la norma de padres, mientras que para los hombres es la norma de amigos.

Lo anterior tiene algunas implicaciones. En primer lugar, la actitud positiva de un adolescente para tener relaciones sexuales, estaría determinada por la percepción que ellos o ellas tienen del nivel de acuerdo de que un o una joven de su misma edad tengan relaciones sexuales, siempre y cuando esta creencia este referida a los o las adolescentes de su mismo sexo. Así por ejemplo, si una joven cree que otras jóvenes iguales a ella no están de acuerdo con tener relaciones sexuales, probablemente disminuirá la probabilidad de acceder a ellas, independientemente que las jóvenes crean que sus pares de sexo masculino si están de acuerdo. Así mismo, esta probabilidad aumenta en las mujeres, si sus iguales de sexo femenino están de acuerdo con las relaciones sexuales en la adolescencia, independientemente de que ellas crean que sus pares de sexo masculino no estén de acuerdo. Lo mismo sucedería en el caso de los jóvenes hombres.

En segundo lugar, el sexo de los y las adolescentes también determina una diferencia en la norma social subjetiva, ya que como ya se mencionó, para las jóvenes es más importante lo que piensan sus padres, que los amigos, mientras que para los hombres es más importante lo que piensan los amigos que los padres.

Todo lo anterior, implica que el sexo del adolescente marca una diferencia en el algoritmo del modelo a la hora de predecir las decisiones que los y las jóvenes puedan tener en relación con el inicio o no de sus relaciones sexuales, lo que estaría indicando que los programas de prevención de embarazo adolescente deben contemplar el enfoque de género a la hora de su diseño, implementación y evaluación. 
En conclusión, este estudio permitiría afirmar que la actitud positiva que los y las jóvenes tengan hacia las relaciones sexuales en la adolescencia, está determinada por lo que piensan sus pares del mismo sexo. Así mismo, se confirmó que la variable norma moral mejora el poder predictivo de la TAR para predecir la intención para empezar a tener relaciones sexuales en la adolescencia, que la norma social subjetiva de amigos es determinante en la intención de los hombres, mientras que para las mujeres es la norma de padres.

Finalmente, es importante tener las limitaciones del estudio. Dado que se trata de un diseño de tipo observacional, sin manipulación variable, ni control de variables extrañas, es necesario recordar que este tipo de estudios no pueden asegurar relaciones de causalidad entre las variables. Por otra parte, el estudio tomó una muestra intencional en tres colegios de Bogotá (Colombia), ésto limitaría también la generalización de los resultados a la población general de jóvenes.

\section{Referencias}

1. Chalmers H, Aggleton P, Stone N, Ingham R. Dynamic contextual analysis of young people's sexual health; a context specific approach to understanding barriers to, and opportunities for, change. Southampton: DFID at University of Southampton; 2001.

2. Flórez CE, Núñez J. Teenage childbearing in Latinamerican countries. Inter-American Development Bank; Bogotá: CEDE. 2000 Aug. Report No.: R-434.

3. Flórez CE, Núñez J. Teenage Childbearing in Latin American Countries. Bogotá: CEDE; 2002.

4. Flórez CE, Soto V. Fecundidad adolescente y pobreza. Diagnóstico y lineamientos de política; Bogotá: CEDE. 2005.

5. Ministerio de Protección Social de Colombia. Politica nacional de salud publica 2007-2010. Ministerio de Protección Social de Colombia; 2012. Disponible en: http://javeriana.edu.co/redcups/ Politicas_Nacionales_Salud-Colombia_2007-2010.pdf

6. Baker SA, Thalberg SP, Morrison DM. Parents' Behavioral Norms as Predictors of Adolescent Sexual Activity and Contraceptive Use. Adolescence. 1988; 23(90): 265-82.

7. Capaldi DM, Crosby L, Stoolmiller M. Predicting the timing of first sexual intercourse for at-risk adolescent males. Child Development. 1996; 67(2): 344-59.

8. Furman W, Shaffer L. The Role of romantic relationships in adolescence development. En: Florsheim P, editor. Adolescent Romantic Relations and Sexual Behavior: Theory, Research, and Practical Implications. Mahwah, NJ, US: Lawrence Erlbaum Associates Publishers; 2003.

9. Lanctôt N, Smith CA. Sexual activity, pregnancy, and deviance in a representative urban sample of African American girls. Journal of Youth and Adolescence. 2001; 30(3): 349-72.

10 Longmore M, Manning W, Giordano P, Rudolph J. Self-esteem, depressive symptoms, and adolescents' sexual onset. Center for Family and Demographic Research BGO, editor. 2003. Ref. Tipo: Trabajo no publicado.
11 Meier A. Adolescent sex and subsequent mental health: How sex affects adolescent depression and self-esteem. Wisconsin: Universidad de Wisconsin, Center for Demography and Ecology; 2002.

12 Meschke LL, Zweig JM, Barber BL, Eccles JS. Demographic, biological, psychological, and social predictors of the timing of first intercourse. Journal of Research on Adolescence. 2000; 10(3): 315-38.

13 Miller KS, Forehand R, Kotchick B. Adolescent sexual behavior in two ethnic minority groups: A multisystem perspective. Adolescence. 2000;35 (138): 313-33.

14 Forehand R, Gound M, Kotchick B, Armistead L, Long N, Miller K. Sexual Intentions of Black Preadolescents: Associations with Risk and Adaptive Behaviors. Perspectives on Sexual and Reproductive Health. 2005; 37(1): 13-8.

15 Smith C. Factors associated with early sexual activity among urban adolescents. Social Work. 1997; 42(4): 334-46.

16 Welsh D, Grello C, Harper M. When Love Hurts: Depression and adolescent romantic relationships. En: Florsheim P, editor. Adolescent Romantic Relations and Sexual Behavior: Theory, Research, and Practical Implications. Mahwah, NJ: Lawrence Erlbaum Associates Publishers; 2003. p.185-211.

17 Whitbeck LB, Yoder KA, Hoyt DR, Conger RD. Early adolescent sexual activity: A developmental study. Journal of Marriage and the Family. 1999; 61(4): 934-46.

18 Belgrave FZ, Van Oss Marín O, Chambers DB. Cultural, contextual, and intrapersonal predictors of risky sexual attitudes among urban African American girls in early adolescence. Cultural Diversity and Ethnic Minority Psychology. 2008; 6(3): 309-22.

19 Pedersen W, Samuelsen SO, Wichstrøm L. Intercourse debut age: Poor resources, problem behavior or romantic appeal? The Journal of Sex Research. 2003; 40(4): 333-45.

20 Conner M, Norman P. The rol of social cognition in health behaviours. En: Conner M, Norman P, editores. Predicting health behavior. Buckingham, United Kingdom: Open University; 1996. p. 1-22.

21 Fishbein M, Ajzen I. Belief, Attitude, Intention, and Behavior: An Introduction to Theory and Research. Addison-Wesley, Reading, Mass; 1975.

22 Bandura A. Social fundations of thought and action: A social cognitive theory. Nueva Jersey: Prentice-Hall, Englewood Cliffs; 1986.

23 Norman P, Conner M. Predicting health check attendance among prior attenders and non-attenders: the role of prior behaviour in the theory of planned behaviour. Journal of Applied Social Psychology. 1996; 26(11): 1010-26.

24 Ajzen I, Fishbein M. Factors influencing intentions and the intention behavior relation. Nueva York: Human Relations; 1974.

25 Ajzen I, Fishbein M. Understanding atitudes and predicting social behavior. Nueva Jersey: Prentice-Hall, Inc.; 1980.

26 Sparks P, Sheperd R. The role of moral judgements within expectancy-value based attitude-behavior model. Ethics and Behabior. 2002; 12: 299-321.

27 Garcia-Díaz D-M. Factores explicativos de la intención de las adolescentes de tener relaciones sexuales: un análsis a partir de la teoría del comportamiento palneado. Bogotá: Universidad de los Andes; 2005.

28 Gillmore MR, Archibald ME, Morrison DM, Wilsdon A, Wells EA, Hoppe MJ, et al. Teen sexual behavior: Applicability of the Theory of Reasoned Action. Journal of Marriage and Family. 2002; (64): 4-885.

29 Baumer EP, South SJ. Community Effects on Youth Sexual Activity. Journal of Marriage and the Family. 2001; 63(2): 540-54. 
30 Carvajal SC, Parcel GS, Banspach SW, Basen-Engquist K, Coyle KK, Kirby D, et al. Psychosocial predictors of delay of first sexual intercourse by adolescents. Health Psychology. 1999; 18: 443-52.

31 Flórez CE, Vargas E, Henao J, Gonzáles C, Soto V, Kassem D. Fecundidad adolescente en Colombia: incidencia, tendencias y determinantes. Un enfoque de historia de vida. Bogotá: CEDE; 2004.

32 Kinsman SB, Romer D, Furstenberg FF, Schwarz DF. Early sexual initiation: The role of peer norms. Pediatrics. 1998; 102(5): 1185-92.

33 Miller KS, Kotchick B, Forehand R. Adolescent sexual behavior in two ethnic minority samples: The role of family variables. Journal of Marriage and the Family. 1998; 61(1): 85-98.

34 Whitaker DJ, Miller KS, Clark L. Reconceptualizing adolescent sexual behavior: Beyond did they or didn't they? Family Planning Perspectives. 2000; 32(3): 111-24.

35 Scholly K, Katz A, Gascoigne J, Holck P. Using Social Norms Theory to Explain Perceptions and Sexual Health Behaviors of
Undergraduate College Students: An Exploratory Study. Journal Of American College Health. 2005; 53(4): 159-66.

36 Martens M, Page J, Mowry E, Damann K, Taylor K, Cimini D. Differences Between Actual and Perceived Student Norms: An Examination of Alcohol Use, Drug Use, and Sexual Behavior. Journal Of American College Health. 2006; 54(5): 295-301.

37 Hagman B, Clifford P, Noel N. Social Norms Theory-Based Interventions: Testing the Feasibility of a Purported Mechanism of Action. Journal Of American College Health. 2007; 56(3): 293-9.

38 Wellings K, Collumbie M, Slaymaker E, Singh S, Hodges Z, Patel $\mathrm{H}$, et al. Sexual behaviour in context: a global perspective. The Lancet. 2006;368:1706-28.

39 Vargas E, Barrera F. El papel de las relaciones padres-hijos y de la competencia psicosocial en la actividad sexual de los adolescentes. Bogotá: CESO; 2002. 32.

40 Vargas E, Barrera F. Actividad sexual y relaciones románticas durante la adolescencia: algunos factores explicativos. Bogotá: CESO; 2003. 